\title{
Optimization Technique and FE Simulation for Lag Screw Placement in Anterior Column of the Acetabulum
}

\author{
Ruo-feng Tong, Sheng-hui Liao, and Jin-xiang Dong \\ State Key Laboratory of CAD and CG, \\ Department of Computer Science and Engineering, \\ Zhejiang University, China \\ liaoshenhui@zju.edu.cn
}

\begin{abstract}
This paper presents an optimization technique for determining the lag screw placement in the anterior column of the acetabulum, and investigates new method for generating accurate finite-element (FE) model for biomechanics analysis. For prepare once measure, an accurate hemi-pelvis model is reconstructed from the volume-of-interest extracted from computed-tomography (CT) data, and the initial position of the lag screw is determined by traditional manual like method. Then, an objective function, for improving the placement of lag screw, is build by adaptive sampling the weighted distance of screw to the acetabulum boundary according to surgical requirement, and the two end points of the lag screw are modified iteratively to reduce the objective value. 30 hemi-pelvis models are tested by the optimization technique, and the statistical measure data are provided according to new anatomic reference landmarks for clinical use. In the second part, FE method is employed to evaluate the optimization result. To generate accurate and high quality FE model, a semi-automatic FE preprocessor specifically adapted to the pelvis anatomy is developed. The produced volume mesh has a very regular mesh structure and achieves a smooth change of element size transition. The final simulation stress distribution pattern justifies the placement of the lag screw in the anterior column of the acetabulum.
\end{abstract}

\section{Introduction}

Several studies have shown that open reduction and internal fixation of displaced acetabular fractures improve functional and clinical results [1]. Lag screw fixation along the long axis of the anterior column has been recommended for the treatment of transverse and $\mathrm{T}$ type fractures. However, proper placement of a lag screw in the anterior column is challenging because of its unique anatomy, relatively small cross sectional area, and the risk of violation of the hip joint [2]. 


\section{Technology for Determine the Placement of Lag Screw}

\subsection{Previous Work}

There are several approaches proposed in the literature to determine the placement of the lag screw in anterior column of the acetabulum in recent years. Mears and Rubash [3] suggested that a starting point for lag screw fixation of the anterior column should be chosen $2.5 \mathrm{~cm}$ above the roof of the acetabulum, and the screw should be directed parallel to the iliopectineal line. Letournel and Judet 4 advocated an entry point 3 to $4 \mathrm{~cm}$ above the acetabular roof and recommended that the direction of screw placement be controlled visually and by palpation of the iliopectineal eminence with a finger. The study of Ebraheim et al 5] showed that the entry point for lag screw placement along the functional axis of the anterior column in a sagittal plane can be localized intraoperatively by palpating the bony landmarks and measuring a mean distance of $42 \mathrm{~mm}$ posteriorly from the anterior interspinous notch, and $46 \mathrm{~mm}$ from the superior acetabular rim. And the inclination of the screw placement from this starting point should be $90.6 \pm 5.0$ degree in the sagittal plane and $29.0 \pm 4.4$ degree in a transverse plane.

All of the previous approaches were based on clinic experience or measured directly on embalmed pelvis. One of the shortcomings of these methods is the embalmed pelvises are difficult to get, often with poor bone quality, and always have the virus risk. Second, because of the irregular bony structure, manual work is tend to introduce measure error. Third, physical methods usually need to dissect the models, and can measure once only. Fourth, some geometric information, such as some angle, is hard to measure by simple instrument on the physical model.

\subsection{Investigate Optimal Lag Screw Placement}

This paper selects the CT volume as the source data because 3D CT imaging of pelvis patients is routine at present, and can provide accurate data information which is needed for the experimentation.

For prepare once measure, an accurate hemi-pelvis model is reconstructed from the volume-of-interest extracted from CT volume, and the initial position of the lag screw is determined by manual method like paper [5. First, selecting the initial cut as the inferior bony acetabular margin, the model is sectioned at 1 $\mathrm{cm}$ intervals perpendicular to the anterior surface of the anterior column. A line designated $\mathrm{AB}$, is drawn perpendicular to the pelvis brim on the caudal surface of the cross section 1 and $3 \mathrm{~cm}$ superior to the initial cut connecting the (A) lateral bony acetabular margin and the (B) pelvic brim, and the midpoint for this line is determined. A second perpendicular line, $\mathrm{CD}$, is drawn on the midpoint of line $\mathrm{AB}$. The midpoint of line $\mathrm{CD}$ is determined to yield the midpoint (X) of the two cross sections of the anterior column, which are on the functional axis of the anterior column of the acetabulum. Then, the projection points of the functional axis on the outer table of the iliac wing and the pubic bone are calculated. Such as Fig 1 shows. 


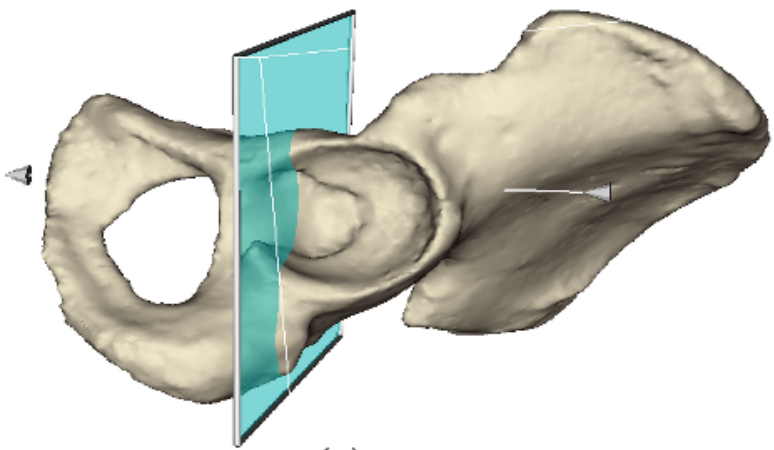

(a)

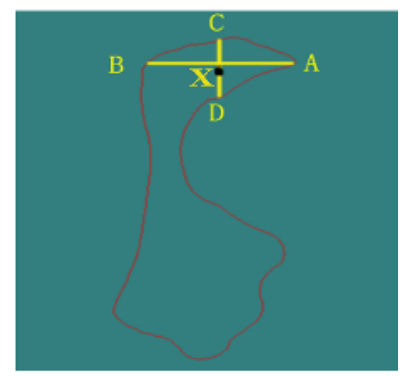

(b)

Fig. 1. The initial placement of the lag screw

While, as the configuration of the entire anterior column is irregular and has a curved true axis, the functional axis determined by only two cross sections can not guarantee for the globe optimal placement. This paper designs an objective function to improve the placement of lag screw in the anterior column of the acetabulum.

Our consideration is based on the clinical requirement: to avoid the violation of the acetabulum and cortical penetration, the distance from the lag screw to the boundary of the anterior column of the acetabulum should as large as possible.

Start from the initial placement of the screw, the algorithm adaptively samples the weighted distance of screw to the acetabulum boundary. Assume the current number of sample cross sections, which are perpendicular to the lag screw, is N. For each cross section $i, i=1,2 \ldots N$, the algorithm calculates the shortest vector from the center of the screw $\boldsymbol{P}_{i}$ on the section plane to the acetabulum boundary:

$$
\boldsymbol{S} \boldsymbol{V}_{i}=\min \left\{\left(\boldsymbol{V}_{i}\right)_{\alpha}\right\}, 0 \leq \alpha \leq 359
$$

where $\left(\boldsymbol{V}_{i}\right)_{\alpha}$ is an function of $\boldsymbol{P}_{i}$ and searches in the extracted volume-of-interest directly rather than done a significant number of intersection calculations with the discrete surface model. Define the weight value as inverse proportion to the length of the shortest vector: $\frac{c}{\left\|\boldsymbol{S} \boldsymbol{V}_{i}\right\|}$, where $c$ is a constant. Then the hint moving vector of section $i$ is:

$$
\boldsymbol{D}_{i}=\frac{c}{\left\|\boldsymbol{S} \boldsymbol{V}_{i}\right\|}\left(-\frac{\boldsymbol{S} \boldsymbol{V}_{i}}{\left\|\boldsymbol{S} \boldsymbol{V}_{i}\right\|}\right)=-c \frac{\boldsymbol{S} \boldsymbol{V}_{i}}{\left\|\boldsymbol{S} \boldsymbol{V}_{i}\right\|^{2}}
$$

The globe objective function can be constructed as the sum of these $\mathrm{N}$ hint moving vectors' norm:

$$
\operatorname{Obj}=\sum_{i=1}^{N}\left\|D_{i}\right\|
$$

It is clear that when $O b j$ reduces to the minimal value, the lag screw get the optimal placement. 
Assume the two end points, which determine the placement of screw, are $\boldsymbol{P}_{\text {start }}=<X_{1}, Y_{1}, Z_{1}>$ and $\boldsymbol{P}_{\text {end }}=<X_{2}, Y_{2}, Z_{2}>$. Then the center position of the screw on each cross section plane is $\boldsymbol{P}_{i}=\frac{r_{2}}{r_{1}+r_{2}} \boldsymbol{P}_{\text {start }}+\frac{r_{1}}{r_{1}+r_{2}} \boldsymbol{P}_{\text {end }}$. This proportion gives us a suggest that the influence of each hint moving vector can be brought to the two end points directly, $\sum_{i=1}^{N} \boldsymbol{D}_{i}=\sum_{i=1}^{N} \frac{r_{2}}{r_{1}+r_{2}} \boldsymbol{D}_{i}+\sum_{i=1}^{N} \frac{r_{1}}{r_{1}+r_{2}} \boldsymbol{D}_{i}$, rather than do a numerical derivation. So, the moving direction of two end points can be defined as $\boldsymbol{D}_{\text {start }}=\sum_{i=1}^{N} \frac{r_{2}}{r_{1}+r_{2}} \boldsymbol{D}_{i}$ and $\boldsymbol{D}_{\text {end }}=\sum_{i=1}^{N} \frac{r_{1}}{r_{1}+r_{2}} \boldsymbol{D}_{i}$, and the moving distance can be calculated by a linear search procedure.

Now, the two end points of the lag screw are modified iteratively to reduce the objective value until $\triangle O b j$ reaches a threshold. During the iteration, the number of sample cross sections $\mathrm{N}$ can be adaptively increased until the $\mathrm{CT}$ spacing resolution is reached, this is helpful to prevent local minimal result.

\subsection{Statistical Measure Data}

For convenient clinical use, we investigate new anatomic reference landmarks, especially for the inclination of the functional axis, such as Fig 2 shows. In all, 30 hemi-pelvis models are tested by the optimization technique, and the statistical measure data are listed as follows:

(1) The mean distance FG, between the anterior interspinous notch (F) and the intersection of the perpendicular line from the projection point $(\mathrm{P})$ to the curve line EF connecting the apex of the sciatic notch (E) with the anterior interspinous noth $(\mathrm{F})$, is $39.48 \pm$ standard deviation of $2.12 \mathrm{~mm}$.

(2) The mean distance EG, between apex of the sciatic notch (E) and the intersection of the perpendicular line from the projection point $(\mathrm{P})$ to the line $\mathrm{EF}$, is $41.38 \pm 2.23 \mathrm{~mm}$.

(3) The mean distance PG, between the projection point $(\mathrm{P})$ and its perpendicular intersection on the curve line $\mathrm{EF}$, is $16.33 \pm 3.31 \mathrm{~mm}$.

(4) The mean distance GH, between the perpendicular intersection point on curve line EF $(\mathrm{G})$ and the superior rim of acetabulum $(\mathrm{H})$, is $25.65 \pm 0.82 \mathrm{~mm}$.

(5) The mean length of the screw for anterior column fixation is 104.24 \pm $4.63 \mathrm{~mm}$.

(6) For the inclination of the functional axis of the anterior column, we use the angle $\angle S P A$, between the axis and the line PA connecting the projection point (P) with the posterior inferior iliac spine $(\mathrm{A})$, which is $41.52 \pm 0.92$ degree. And the angle $\angle S P Q$, between the axis and the line $\mathrm{PQ}$ connecting the projection point $(\mathrm{P})$ with the point $(\mathrm{Q})$ on the radial line $\mathrm{GP}$ as we found the point $\mathrm{G}, \mathrm{P}$ and $\mathrm{Q}$ are almost on a common plane, which is $35.35 \pm 1.12$ degree.

\section{$3 \quad$ Finite Element Simulation}

To evaluate the optimization placement result, the FE method is used for predicting the biomechanical behavior of the pelvis with the lag screw in the anterior column of the acetabulum. However, creating an accurate FE pelvis model is not 


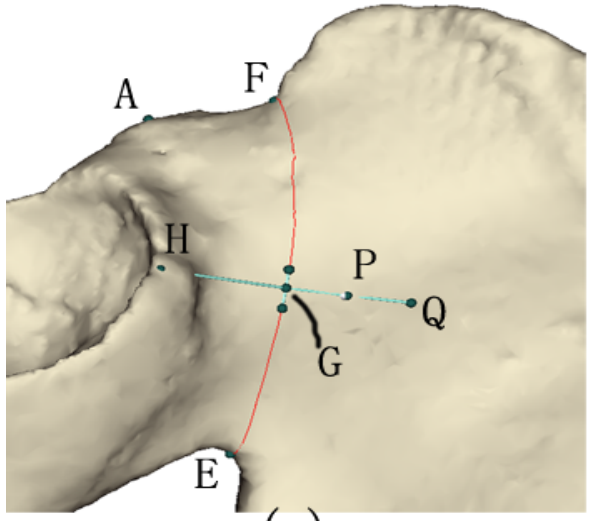

(a)

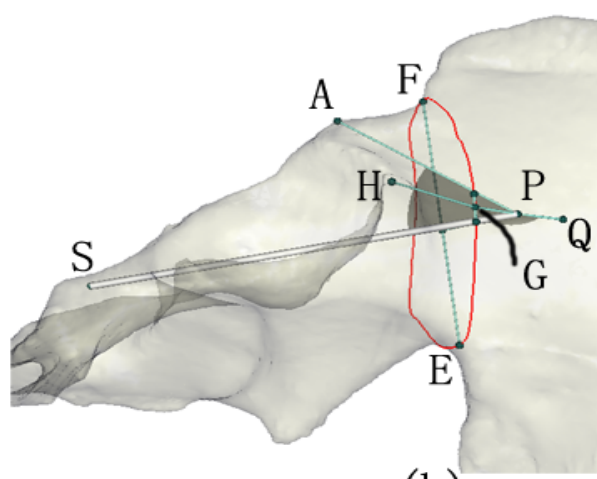

(b)

Fig. 2. The distance and angle measurements

an easy work, which has lots of complex anatomical features, such as acetabulum, foramen obturaturm, and so on.

For general automatic volume mesh generation, tetrahedral is by far the most common form. Most current techniques can fit into one of three main categories: the octree method [6], Delaunay triangulation [7, 8] 9] [10] and the advancing front approach [11. While, general volume meshing methods do not work well to create a satisfactory FE pelvis model efficiently, as they did not consider the patient-specific shape configuration.

There are many FE modelling work specially for biomechanical analysis proposed in the literature in recent years, most of them extracted geometric information from embalmed physics model or CT data at a serial of parallel cross section, and the generated meshes align inherently with the orthogonal plane, which does not properly account for the preferential orientation feature of the physical model.

This paper presents a semi-automatic FE preprocessor to extract the crucial geometry of the highly irregular bony structure of the hemi-pelvis, to input into downstream process for the development of FE model. The mesh developed herein has to be well-represented in terms of original geometry as well as achieving a high quality of mesh as poor representation and quality of finite element would lead to inaccurate results.

\subsection{Semi-automatic FE Preprocessor to Generate Volume Mesh}

The core consideration of our algorithm is to use curve cut surface, which properly account for the preferential orientation feature of the physical model, to extract the crucial geometry. This is accomplished by our semi-automatic FE preprocessor.

To control the placement of these curve cut surfaces, the preprocessor first creates pairs of control spline by setting several anatomical landmarks interactively, such as these dark blue splines in Fig 3 shows. The shape of the curve cut 


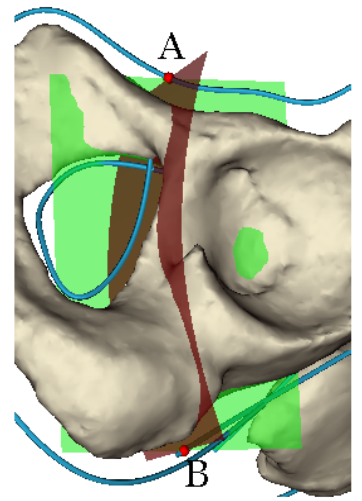

(a)

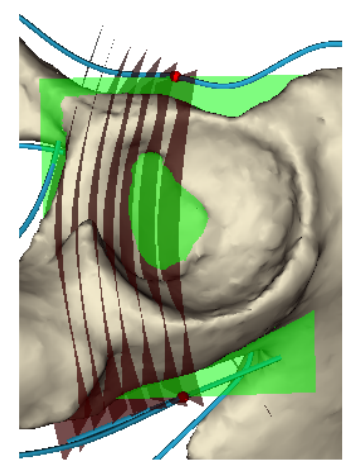

(b)

Fig. 3. The curve cut Surfaces

surface is determined as follows: for each selected pair points A and B on the control splines, a direction plane is determined by the axis $\mathrm{AB}$ and the average direction of the two control splines at the point $\mathrm{A}$ and $\mathrm{B}$, such as the green plane in Fig 3(a) shows; then, the initial cut surface, with $A B$ as its axis, is generated perpendicular to the direction plane; the axis $\mathrm{AB}$, which is a B-spline, can be bent arbitrarily on the direction plane, and result to a free bend curve cut surface, such as the red curve cut surface shown in Fig 3. (a).

To create the whole model, some key curve cut surfaces are first determined manually, then the in-between curve cut surfaces are generated automatically by linear interpolation, such as Fig[3(b) shows.

After all of the curve cut surfaces are determined, the sample points on the cut contours are produced by an adaptive scheme. The main factors of the sample distance on the cut contour are: (1) the distance of current point to the adjacent contour; (2) the curvature of local surface; (3) the anatomical features
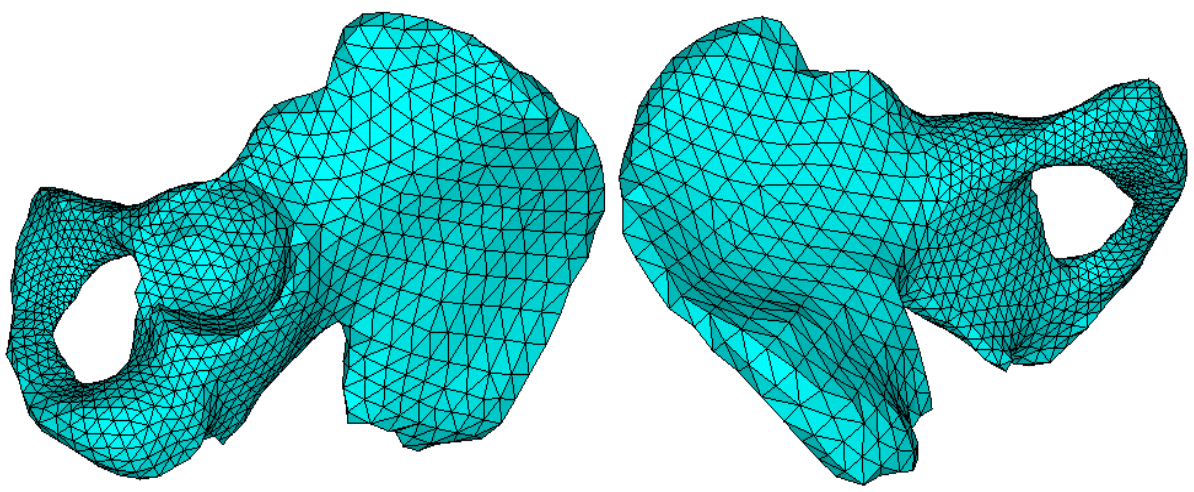

Fig. 4. The result hemi-pelvis FE model 
of the physical model. Then the surface mesh is created based on these extracted geometric information.

And as the curve cut surfaces are expandable, the FE preprocessor creates additional insertion points by $2 \mathrm{D}$ triangulation, then mapped back to the curve cut surfaces. Finally, the volume mesh is created using the surface mesh together with these insertion points by 3D Delaunay triangulation. And the bony material properties are assigned corresponding to the Hounsfield value of CT. The detailed discussion is ignored because of space limitation.

Fig 4 shows the result, we can see that the finite element model developed from this process has a highly regular mesh structure and achieves a smooth change of element size transition, as well as good representation of its original geometry, which would give a better prediction of its biomechanical response in vivo and in vitro situations.

\subsection{FE Biomechanics Analysis}

Finally, the pelvis model combined with the lag screw in the anterior column of the acetabulum is created, such as Fig 5 (a) shows. Axis direction loading is added on the screw nailhead, and the simulation stress distribution has a uniform pattern, which justifies the placement of the lag screw in the anterior column of the acetabulum, such as Fig 5 (b) shows.

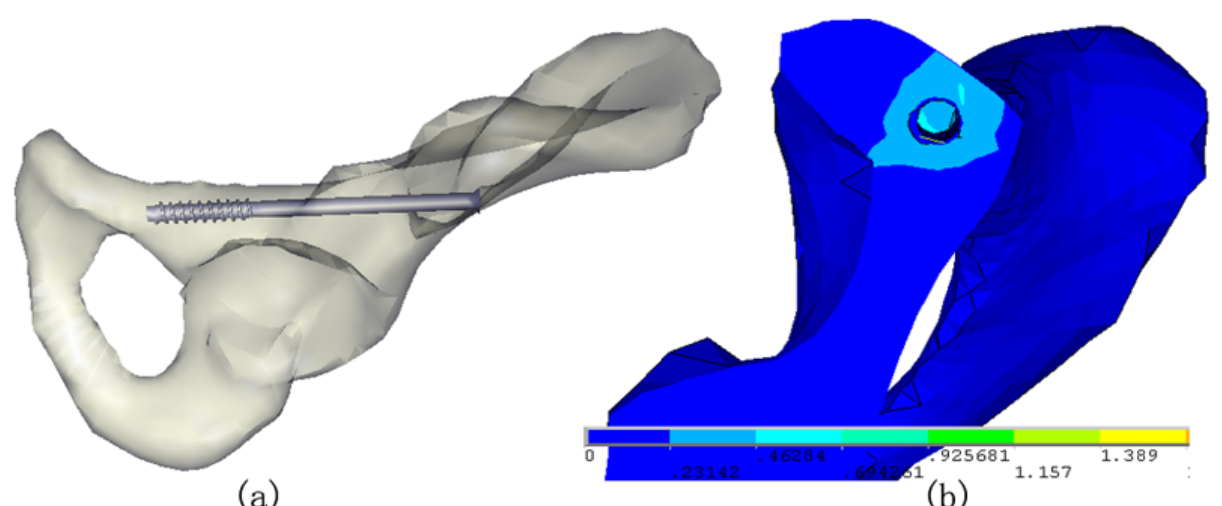

Fig. 5. The combined model and simulation stress distribution pattern

\section{Conclusion}

We have presented an automatic optimization technique for improving the lag screw placement in the anterior column of the acetabulum. The objective function is constructed directly based on the clinical requirement, and the adaptive iteration scheme is bound to find the globe minimal value. In addition, a particular convenient FE preprocessor which take advantages of the curve cut surface, 
is developed. And the result model has a mesh structure of high quality and preserves the original geometry feature, which is of the essence in the study of accurate biomechanics Analysis. The method here can be applied easily to create other finite element volume meshes. We intend to do some advanced biomechanical study in the near future and improve robustness of the algorithm.

\section{Acknowledgments}

This project was supported by the Natural Science Foundation (No.M603129) of Zhejiang Province, China.

\section{References}

1. Schopfer A, Willett K. Powell J, Tile M: Cerclage wiring in internal fixation of acetabular fractures. J Orthop Trauma 7:236-241, 1993.

2. Anglen JO, DiPasquale T: The reliability of detecting screw penetration of the acetabulum by intraoperative auscultation. J Orthop Trauma 8:404-408, 1994.

3. Mears DC, Rubash HE: Techiques of Internal Fixation. In Mears DC, Rubash HE (eds). Pelvic and Acetabular Fractures. Thorofare, NJ, Slack 299-318, 1986.

4. Letournel E, Judet R: Operative Treatment of Specific Type of Fractures. In Letournel E, Judet R (eds). Fractures of the Acetabulum. Ed 2. Berlin, SpringerVerlag 442-447, 1993.

5. Ebraheim NA, Xu R, Biyani A, Benedetti JA.: Anatomic basis of lag screw placement in the anterior column of the acetabulum. Clin Orthop Relat Res. 1997 Jun;(339):200-5.

6. Mark S. Shephard and Marcel K. Georges, "Three-Dimensional Mesh Generation by Finite Octree Technique". International Journal for Numerical Methods in Engineering, 1991,vol 32, pp. 709-749.

7. H. Borouchaki, F. Hecht, E. Saltel and P. L. George. "Reasonably Efficient Delaunay Based Mesh Generator in 3 Dimensions", Proceedings 4th International Meshing Roundtable, pp.3-14, October 1995.

8. N. P. Weatherill and O. Hassan. "Efficient Three-dimensional Delaunay Triangulation with Automatic Point Creation and Imposed Boundary Constraints". International Journal for Numerical Methods in Engineering, 1994,vol 37, pp.2005-2039.

9. S. Rebay. "Efficient Unstructured Mesh Generation by Means of Delaunay Triangulation and Bowyer-Watson Algorithm", Journal Of Computational Physics, 1993,vol. 106, pp.125-138.

10. David L. Marcum and Nigel P. Weatherill. "Unstructured Grid Generation Using Iterative Point Insertion and Local Reconnection", AIAA Journal, September, 1995,vol 33, no.9, pp.1619-1625.

11. R. Lohner. "Progress in Grid Generation via the Advancing Front Technique", Engineering with Computers,1996, vol 12, pp.186-210. 